$A B$

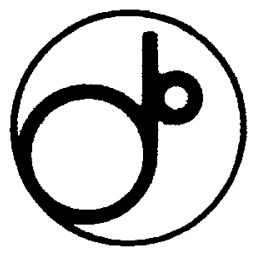

KEK Preprint 95-119

October 1995

H

\title{
Measurement of Charm Production in Two-photon Processes using Inclusive Lepton Events at TRISTAN
}

AMY Collaboration

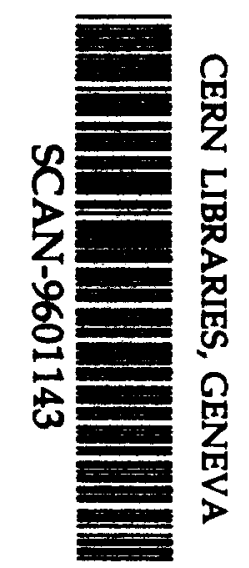

509605

Submitted to Phys. Lett. B. 
National Laboratory for High Energy Physics, 1995

KEK Reports are available from:

Technical Information \& Library

National Laboratory for High Energy Physics

1-1 Oho, Tsukuba-shi

Ibaraki-ken, 305

JAPAN

Phone: 0298-64-5136

Telex: $\quad 3652-534$

(0)3652-534

Fax: $\quad$ 0298-64-4604

Cable: $\quad$ KEK OHO

E-mail: Library@kekvax.kek.jp (Internet Address) 


\title{
Measurement of charm production in two-photon processes using inclusive lepton events at TRISTAN
}

\section{AMY Collaboration}

T.Aso ${ }^{a}$, H.Miyata ${ }^{a}$, T.Sasaki ${ }^{c}$, K.Miyano ${ }^{a}$, N.Nakajima ${ }^{a}$, K.Ohkubo ${ }^{a}$, M.Oyoshi ${ }^{a}$, M.Sato ${ }^{a}$, M.Shirai ${ }^{a}$, N.Takashimizu ${ }^{a}$, Y.Yamashita ${ }^{b}$, K.Abe $^{c}$, Y.Fujii ${ }^{c}$, Y.Kurihara ${ }^{c}$, M.H.Lee $^{c}$, F.Liu $^{c}$, A.Maki ${ }^{c}$, T.Nozaki ${ }^{c}$, T.Omori ${ }^{c}$, H.Sagawa ${ }^{c}$, Y.Sakai ${ }^{c}$, Y.Sugimoto ${ }^{c}$, Y.Takaiwa ${ }^{c}$, S.Terada ${ }^{c}$, P.Kirk ', T.J.Wang ' , A.Abashian ', K.Gotow ' , L.Piilonen', S.K.Choi ', C.Rosenfeld ", L.Y.Zheng ${ }^{h}$, R.E.Breedon ${ }^{i}$, Winston Ko ${ }^{i}$, R.L.Lander ${ }^{i}$, J.Rowe ${ }^{i}$, J.R.Smith ${ }^{i}$, S.Kanda $^{j}$, S.L.Olsen ${ }^{j}$, K.Ueno ${ }^{j}$, R.Tanaka ${ }^{k}$, F.Kajino ', S.Behari ${ }^{m}$, H.Fujimoto ${ }^{m}$, S.Kobayashi ${ }^{m}$, A.Murakami $^{m}$, S.K.Sahu ${ }^{m, t}$, M.Yang ${ }^{m}$, S.Matsumoto ${ }^{n}$, S.Schnetzer ${ }^{\circ}$, A.Bodek ${ }^{p}$, B.J.Kim ${ }^{p}$, J.S.Kang ${ }^{q}$, D.Y.Kim ${ }^{\natural}$, K.B.Lee ${ }^{q}$, H.S.Ahn ${ }^{r}$, S.K.Kim ${ }^{r}$, S.S.Myung ${ }^{r}$, and D.Son *

- Niigata University, Niigata 950.21, Japan

- Nihon Dental College, Niigata 951, Japan

c KEK, National Laboratory for High Energy Physics, Ibaraki 305, Japan

d Louisiana State University, Baton Rouge, LA 70803, USA

e Institute of High Energy Physics, Beijing 100039, China

$f$ Virginia Polytechnic Institute and State University, Blacksburg, VA 24061, USA

- Gyeongsang National University, Chinju 660-701, South Korea

h University of South Carolina, Columbia, SC 29208, USA

- University of California, Davis, CA 95616, USA

'University of Hawaii, Honolulu, HI 96822, USA

* Japan Synchrotron Radiation Research Institute, Hyogo, 679-41, Japan

'Konan University, Kobe 658, Japan

m Saga University, Saga 840, Japan

"Chuo University, Tokyo 112, Japan

- Rutgers University, Piscataway, NJ 08854, USA

P University of Rochester, Rochester, NY 14627, USA

Gorea University, Seoul 196.701, South Korea

- Seoul National University, Seoul 151-742, South Korea

- Kyungpook National University, Taegu 702-701, South Korea

- National Taiwan University, Taipei,10764, Taiwan, R.O.C.

\begin{abstract}
Measurements of open charm production in photon-photon collisions made with the AMY detector at TRISTAN are reported. Charmed hadrons were identified by detecting the high momentum muons or electrons from their semileptonic decays. The data sample corresponds to an integrated luminosity of $275 \mathrm{pb}^{-1}$ at an average center of mass energy of $58 \mathrm{GeV}$. Results are presented in the form of cross sections of inclusive leptons from charm for both muons and electrons. The measured cross section is 1.8 standard deviations higher than theoretical predictions based on the direct and photongluon fusion process, where the mass of charm quark is assumed to be 1.6 $\mathrm{GeV}$.
\end{abstract}




\section{Introduction}

Cross section measurements of the pair production of charm quarks in untagged photon-photon collisions at PEP and PETRA experiments are higher than predictions based on the Born approximation for the direct quark-parton model (QPM) process $\gamma \gamma \rightarrow c \bar{c}[1]$. Recently, the theoretical prediction has been improved by the inclusion of radiative QCD corrections and contributions from resolved photon processes [2]. ("Resolved photon interactions" are hard scattering processes involving the partonic structure of the photon, i.e. the quarks and gluons in the photon [3].) With these inclusions, the theoretical predictions are consistent with the latest experimental results for $D^{*}$ production at PETRA/PEP [2].

More recently, the VENUS and TOPAZ experiments at TRISTAN have reported measurements of charm production in two-photon processes $[4,5,6]$. The TOPAZ results, using both inclusive electron events and $D^{*}$ events, indicate an excess of high transverse momentum of $D^{*}$ s, although both the TOPAZ and VENUS results at low transverse momentum are consistent with the predictions.

Theoretical predictions for charm production are less ambiguous than those for lighter quarks. Among the many different resolved photon processes that contribute to light quark production, only the photon-gluon fusion process gives a sizable contribution to charm production [2]. At TRISTAN energies, the contribution from twiceresolved photon processes to charm production are negligibly small. Moreover, vector meson dominance processes (VMD) [7] are also negligible, and theoretical ambiguities associated with the $P_{t}^{m i n}$ parameter separating the soft and hard scattering processes do not occur. Furthermore, in the high transverse momentum region, the photon-gluon fusion process is largely suppressed and ambiguities relevant to the photon-gluon fusion process, such as the gluon density in the photon, are less important. Similarly, the uncertainty in the charm quark mass has a small effect on the predicted high transverse momentum cross section.

Here, we report a measurement of the cross section for open charm production in untagged two-photon collisions using the AMY detector at TRISTAN. Charmed quarks were identified by their semileptonic decay to high momentum muons or electrons.

We have analyzed a data sample corresponding to an integrated luminosity of 275 $\mathrm{pb}^{-1}$ and have observed more inclusive high momentum leptons than any of the other TRISTAN experiments. Our higher statistics indicate some excess over theoretical predictions at high transverse momenta.

\section{Monte Carlo simulation}

The experimental results are compared with the theoretical predictions using Monte Carlo simulations. Events for both the direct and the photon-gluon fusion process were generated with a Monte Carlo program based on the BASES and SPRING packages [8] and processed though a program that simulates the AMY detector. We take the charm mass to be $1.6 \mathrm{GeV}$.

The direct process is modeled with a QPM event generator that incorporates all six first-order diagrams [9]. Resolved photon events are generated according to the 
formulae given in Ref [3]. Here, three different parameterizations-LAC1 [10], DG [11], and WHIT4 [12]-are used for the parton density in the photon.

The quarks are fragmented via the Lund string-fragmentation scheme in the JETSET7.3 program [13]. The direct process is evolved using a parton shower scheme. The fragmentation function of Peterson et al [14],

$$
f(z)=\frac{N}{z}\left(1-\frac{1}{z}-\frac{\epsilon_{c}}{1-z}\right)^{-2}
$$

is used to describe the fragmentation of charm quarks. Here, $z=\left(E+p_{\|}\right)_{\text {hadron }} /(E+$ $\left.p_{\boldsymbol{l}}\right)_{\text {quark }}, N$ is a normalization constant, and $\epsilon_{c}$ is the fragmentation parameter for charm quarks. We use $\epsilon_{c}=0.047$ [15]. The branching ratios for semileptonic decays of charmed mesons in the LUND decay table were adjusted to match the August 1994 update of the PDG Data Base [16]. The details of the Monte Carlo simulations are described in Ref [17].

\section{Untag event selection}

The data used in this analysis were taken with the AMY detector [18] in the 1990 through 1994 run periods. The center of mass energies range from 57.6 to $59.7 \mathrm{GeV}$ with a luminosity-weighted average of $\sqrt{s}=58 \mathrm{GeV}$. The total data sample corresponds to an integrated luminosity of $275 \mathrm{pb}^{-1}$.

In the selection of inclusive lepton events, we first impose the following untag event criteria:

1) There must be five or more charged tracks originating from within $r=4 \mathrm{~cm}$ and

$|z|=10 \mathrm{~cm}$ of the interaction point, with two or more of these having

$|p| \geq 0.75 \mathrm{GeV} / c$ and at least one having $p_{t} \geq 1.0 \mathrm{GeV} / c$.

2) The net charge of the good tracks must have an absolute value of 2 or less.

3) The net momentum transverse to the beam direction must be $\leq 5.0 \mathrm{GeV} / c$.

4) The visible invariant mass $W_{v 1 s}$ must lie between 4 and $20 \mathrm{GeV}$. Here, the calculation of $W_{v i s}$ includes both charged and neutral particles measured by the barrel tracker (CDC), the barrel calorimeter (SHC), and the endcap calorimeter (ESC).

5) There are no clusters with energy exceeding $0.25 E_{\text {beam }}$ in the electromagnetic calorimeters (SHC , ESC).

Requirement 1) is applied to ensure good trigger efficiency and to reject QED-type events such as $e^{+} e^{-} \rightarrow e^{+} e^{-} \tau^{+} \tau^{-}$. Requirements 2) and 3) reduce the number of events coming from the interactions of beam particles with the residual gas in the vacuum chamber (beam-gas events). Requirement 4) suppresses backgrounds from single-photon hadronic annihilations. Finally, 5) ensures that the two interacting photons were almost real (anti-tag condition). This condition limits the corresponding polar angle for the scattered electron to be $|\cos \theta| \geq 0.97$. Our "untag event sample" contains the 25820 events that pass the above cuts. 


\section{Inclusive muon analysis}

\subsection{Muon selection}

We select muons in the momentum range $2.0 \leq p \leq 5.0 \mathrm{GeV} / c$. They are identified as charged tracks in the CDC that match to hits in any three of the four muon chamber(MUO) layers covering $|\cos \theta|<0.74$. To reduce backgrounds from hadron fakes, we require that the matching distance between the hits and the extrapolated track be less than twice the mean deviation expected from multiple Coulomb scattering. Most cosmic ray events are rejected by requiring that the muon scintillation counter time relative to the beam crossing is in the range $0<t_{\mu}<35 \mathrm{~ns}$. The remaining cosmic ray events are rejected by a visual scan. The efficiency of muon identification is estimated by a Monte Carlo simulation and found to be $40 \%$.

A total of 32 events from the untag event sample satisfy the inclusive muon criteria. No events containing two or more muons were found.

\subsection{Muon backgrounds}

Background from multihadronic single-photon annihilation is estimated to be $1.9 \pm 1.3$ events from a Monte Carlo simulation of this process using the JETSET7.3 generator [13]. Contaminations from $e^{+} e^{-} \rightarrow e^{+} e^{-} \tau^{+} \tau^{-}$and $e^{+} e^{-} \rightarrow e^{+} e^{-} b \bar{b}$ proceses are estimated to be $2.4 \pm 1.4$ and $1.2 \pm 0.2$ events, respectively, from Monte Carlo simulations of these processes.

In bonafide two-photon events, the fake muon signal arises principally from hadron showers in the hadron filter, where the debris reaches the muon chamber ("punchthrough"), or from decays-in-flight of $\pi^{ \pm}$and $K^{ \pm}$mesons to muons that reach the muon chamber ("decay"). These backgrounds were studied by two methods.

1) Monte Carlo simulation

The backgrounds due to decays-in-flight and punchthrough of hadrons were estimated from Monte Carlo event samples for $e^{+} e^{-} \rightarrow e^{+} e^{-}+$hadrons. The amount of background estimated by Monte Carlo was normalized to the data by demanding that the number of Monte Carlo tracks match the number of observed tracks in the untag event sample.

2) Analytical calculation

The background level was also estimated by calculating the probabilities of decay and punchthrough for each charged particle in the untag event sample, using a momentum-dependent analytical formula for the decay and punchthrough probabilities [19]. This calculation did not include the effect of the matching distance requirement.

If, in the Monte Carlo simulation, the matching distance of the MUO hits with an extrapolated CDC track is relaxed to $|d| \leq 1 \mathrm{~m}$ the estimated numbers of background events by these independent methods are consistent within their errors (Fig. 1). When the tighter, two standard deviation matching requirement is imposed in the Monte Carlo simulation, the background due to fake muons was determined to be $8.0 \pm 1.1$ events. 


\subsection{Summary of muon analysis}

After subtracting the background contributions, $18.5 \pm 6.1$ inclusive muon events remain. The results of the muon analysis is summarized in Fig. 2.

\section{Inclusive electron analysis}

\subsection{Electron selection}

A charged track in the momentum range $2.0 \leq p \leq 5.0 \mathrm{GeV} / c$ and within $\pm 2^{\circ}$ (in $\phi$ and $\theta$ ) of the direction of a shower cluster is classified as an electron candidate if the ratio of the shower energy to the track momentum exceeds 0.78 ( 0.77 for charged tracks below $2.5 \mathrm{GeV} / \mathrm{c}$ ), and the longitudinal shower development is consistent with that of an electromagnetic shower. In addition to these cuts, the electron candidate is required to be isolated from the nearest charged track by demanding that the isolation angle between it and the nearest charged track exceeds $6^{\circ}$. Events from the higher-orderQED process, $e^{+} e^{-} \rightarrow e^{+} e^{-} e^{+} e^{-}+\gamma$, are rejected by visual scanning. These criteria were chosen to give good electron efficiency with low pion contamination. The Monte Carlo estimate of the electon identification efficiency is $60 \%$. A total of 49 events from the untag event sample survive as inclusive electron candidates. No events containing two electrons or more or an electron and a muon were found.

\subsection{Electron backgrounds}

Backgrounds from hadronic annihilation (2.8 \pm 1.6 events), $e^{+} e^{-} \rightarrow e^{+} \epsilon^{-} \tau^{+} \tau^{-}(4.0 \pm 1.8$ events), and $e^{+} e^{-} \rightarrow e^{+} e^{-b \bar{b}}$ (1.4 10.2 events) are estimated with the Monte Carlo techniques used for the inclusive muon sample.

The backgrounds in bonafide two-photon events originating from $\gamma$-conversion and $\pi^{0}$ Dalitz decay were estimated by using a Monte Carlo event sample for $e^{+} e^{-} \rightarrow$ $e^{+} e^{-}+$hadrons. Again, we normalize to the same number of tracks for the Monte Carlo event sample and the untag event sample. The combined estimated contribution was $2.8 \pm 0.7$ events.

The backgrounds due to fake electrons were evaluated for the following two possibilities:

1) $\gamma$-hadron overlap

If an electromagnetic shower produced by a $\gamma$ or $\pi^{0}$ is close to a charged track, the charged track could be mistaken as an electron.

An estimate of the background due to overlaps was done using the full untag event sample. A shower cluster in an event was moved artificially to within a 2 degree cone around a charged track and combined with the cluster produced by the track itself. Then the criteria for an electron candidate were applied to the combined cluster. After accounting for the probability that a cluster falls within a 2 degree cone of a charged track, we obtained the $\gamma$-hadron overlap background of $0.5 \pm 0.2$ events.

2) Interacting pion

Pions interacting hadronically in the SHC may produce an energy cluster that 
passes the electron identification criteria. Such interactions are dominated by the charge exchange reaction $\pi^{-}+p \rightarrow \pi^{0}+n$.

This background was estimated using the FLUKA [20] simulation code for the hadronic interaction in conjunction with the AMY detector simulation. In order to examine how well FLUKA simulates hadronic interactions in the AMY detector, we compared the $E / p$ distributions of hadrons in real events with those in Monte Carlo simulated events for single-photon annihilation processes. The results shown in Fig. 3 indicate that FLUKA reproduces the experimental data rather well.

We generated pions with the same momentum and angular distributions as those of the hadrons in the untag event sample and simulated their interactions in the SHC with FLUKA. From these we determine a probability of $4.2 \%$ for a pion to be identified as an electron. Assuming all charged particles in the untag event sample are pions gives an estimate of the interacting pion contamination of the electron sample of $15.4 \pm 0.7$.

\subsection{Summary of electron analysis}

After subtracting the background contributions, we are left with $22.1 \pm 7.5$ inclusive electron events. The electron selection is summarized in Fig. 2.

\section{Cross section for inclusive leptons from charm}

In order to compare the results directly with theoretical predictions, we corrected the results for the limited acceptance to obtain cross sections for inclusive leptons from charm. To ensure good lepton identification, the experiment limits the momenta to $2.0 \leq p \leq 5.0 \mathrm{GeV} / c$ and the polar angles to $|\cos \theta| \leq 0.74$ (0.73) for muons (electrons) to ensure good performance of the lepton identification. Thus, the observed leptons have a minimum transverse momentum relative to the beam line of $p_{t} \geq 1.5$ $\mathrm{GeV} / c$. According to the Monte Carlo simulations of the direct and resolved photon processes, this corresponds to a minimum transverse momentum of the parent $D$-meson $\left(D^{*}, D, D_{s}^{*}\right.$, and $\left.D_{s}\right)$ of $P_{t} \geq 2.0 \mathrm{GeV} / c$ (see Fig. 4). The acceptances for inclusive muon and electron events, determined from these same Monte Carlo simulations, are $14.7 \%$ and $20.1 \%$, respectively.

The detector has no redundant trigger for untagged two-photon events, so the trigger efficiency must also be estimated from the Monte Carlo simulation. The primary trigger for the selected events was derived from track segments in the CDC with some energy deposition in the SHC. We estimate the efficiency for this trigger by combining the track trigger efficiency, as estimated from Monte Carlo trigger simulator, and the SHC energy trigger efficiency, as measured from the data. The overall trigger efficiency is determined to be $89.6 \%$, which is the ratio of the trigger efficiency of the most dominant trigger to the event fraction of the dominant 'rigger in the experiment.

The measured partial cross sections for inclusive leptons from charm $\sigma\left(e^{+} e^{-} \rightarrow\right.$ $\left.e^{+} e^{-l^{ \pm}} X\right)$ are:

$\begin{array}{lll}0.53 \pm 0.16(\text { stat. }) \pm 0.09(\text { syst. }) \mathrm{pb} & \left(2.0 \leq p_{\mu} \leq 5.0 \mathrm{GeV} / c,\right. & |\cos \theta| \leq 0.74) \\ \text { and } \quad 0.46 \pm 0.14(\text { stat. }) \pm 0.09(\text { syst. }) \mathrm{pb} & \left(2.0 \leq p_{e} \leq 5.0 \mathrm{GeV} / c,\right. & |\cos \theta| \leq 0.73)\end{array}$


for inclusive muon and electron events, respectively.

The systematic errors in the measurement of the cross section arise from the luminosity determination, the trigger efficiency estimation, the background subtraction, and the determination of the acceptance correction.

The uncertainty in the luminosity determination was $1.5 \%$.

The error in the trigger efficiency was estimated to be $4.9 \%$ by observing the variation in the trigger efficiency when changing the criteria of the most dominant trigger by combining several track triggers.

The Monte Carlo statistical errors for the determination of background levels from the $e^{+} e^{-} \rightarrow e^{+} e^{-} \tau^{+} \tau^{-}, e^{+} e^{-} \rightarrow e^{+} e^{-} b \bar{b}$, and hadronic annihilation processes are treated as systematic uncertainties. These are 8.9 and $9.6 \%$ for the inclusive muon and electron events, respectively.

In the fake muon determination, the uncertainty in the value of the cross section in the GVMD model used to generate $e^{+} e^{-} \rightarrow e^{+} e^{-}+$hadrons was taken into account. The systematic error taken to be the variation of the results when the value of cross section was changed by $50 \%$. We compared the number of fake muons estimated from analytical calculation with that of Monte Carlo simulation. The difference in the results from these independent two methods was taken as an additional systematic error.

In the determination of the contamination from fake electrons, there is an uncertainty associated with the choice of Monte Carlo models for simulation of hadron interaction. The FLUKA Monte Carlo simulation code was used for the estimation of this background. We evaluated the systematic error of FLUKA using the $E / p$ distributions of charged tracks in hadronic annihilation process. The $E / p$ distributions obtained by FLUKA were compared with that of experimental data. We modified the $E / p$ distributions of FLUKA by one standard deviation from the default distributions and took the variation of the efficiency of hadrons that pass our electron cuts as a measure of this systematic error.

The total systematic uncertainty in the determinations of the fake lepton background are the quadrature sums of the above-listed errors together with the errors associated with the Monte Carlo statistics. The results are 8.1 and $8.0 \%$ for inclusive muon and electron events, respectively

The acceptance correction factor was recalculated by changing cuts for the acceptance boundary of leptons by amounts corresponding to the experimental resolutions, by using different parton distribution function ( $\mathrm{LACl}$ and $\mathrm{DG}$ ), and by varying the branching ratios of the charmed mesons, either the August 1994 PDG tables or the JETSET7.3 default values, in the LUND decay table as the Monte Carlo inputs. The systematic error due to acceptance correction was estimated to be $12 \%$ for both inclusive muon and electron events.

The quadratic sum of the systematic errors are 17 and $19 \%$ for inclusive muon and electron events, respectively; about half of the statistical errors.

\section{Discussion}

The measured cross sections and theoretical predictions are summarized in Table 1. Fig. 5 shows our measurements compared with theoretical predictions of the incoherent 
sum of the direct and photon-gluon fusion processes. In these calculations, we used the LAC1, DG, and WHIT4 model calculations for the gluon density.

In this analysis, next-to-leading order(NLO) QCD corrections are applied to the direct and resolved-photon charm production processes. In determining this correction, we removed the effects of hard gluon radiation from the total NLO correction[6, 21], since these are already included in our Monte Carlo generator. The dominant correction to the direct process is found to be the gluon exchange diagram; a $W_{\gamma \gamma}$-dependent correction is obtained [2], where $W_{\gamma \gamma}$ is the invariant mass of the $\gamma \gamma$ system. The correction for the resolved photon process is dominated by the $\gamma q \rightarrow c \bar{c} q$ process and is given to a good approximation by the function:

$$
N\left(0.48 p_{T}^{c}+0.52\right) \text {, }
$$

where $p_{r}^{c}$ is the transverse momentum of the charm quark in $\mathrm{GeV} / c$ and $N$ is the normalization factor that is determined to be 1.07 from fits of the total NLO cross section to resolved photon process measurements. Here, the charm quark mass $\left(m_{c}\right)$ and the renormalization scale $(\mu)$ are taken as $1.6 \mathrm{GeV}$ and $\sqrt{2} m_{c}$, respectively.

The results of the calculations listed in Table 1 indicate that the resolved photon contribution process is only 10 to $30 \%$ of the direct process, depending on the parton density function used for the photon. In these calculations, resolved photon processes give a softer charm-quark momentum distribution than that of direct process, which correspondingly leads to a softer lepton momentum distribution. This reduces the contribution of the resolved photon process for the lepton momentum region covered by this experiment. Consequently, the sum of the direct and the resolved photon process does not change significantly for the quite different gluon distribution functions of LAC1, DG, and WHIT4. The sum of the muon and electron results is 1.8 standard deviation larger than the prediction with the WHIT4 distribution function (Fig. 5); this excess becomes somewhat larger if the LAC1 or DG densities are used.

We have examined the effects of the mass and fragmentation parameter of the charm quark on the theoretical prediction of the cross sections. A lower mass gives a higher cross section and vice versa. The effect is more pronounced in the low momentum region. In the high momentum region covered by this measurement, the effect of lowering the charm quark mass from 1.6 to $1.3 \mathrm{GeV}$ is about a $10 \%$ increase in the cross section. The cross section calculations using a charm quark mass of $1.3 \mathrm{GeV}$ are summarized in Table 2.

Using a harder fragmentation function for the transition of the charm quark to hadrons, which corresponds to a smaller fragmentation parameter $\epsilon_{c}$, makes the predicted cross sections larger, since the transverse momenta of the parent $D$ is correlated with that of the lepton. We changed the value of fragmentation parameter to 0.028 from the chosen value of 0.047 , according to the ALEPH group's measurement [15]. This increases the cross sections by 15 and $12 \%$ for the inclusive muons and electrons, respectively.

The TOPAZ group has measured the inclusive electron cross section as a function of the electron transverse momentum [5]. Since the momentum range and solid angle acceptance of the two experiments differ, the results can not be compared directly. We extrapolate our cross section for inclusive electron from charm to the region covered by TOPAZ using a Monte Carlo simulation of the direct process. For this comparison, the transverse momenta and polar angles of electrons are limited to $1.5 \leq P_{t} \leq 3.0 \mathrm{GeV} / \mathrm{c}$ 
and $|\cos \theta| \leq 0.77$, respectively. Our extrapolated cross section is about $50 \%$ smaller than that of TOPAZ, but consistent within one standard deviation.

\section{Summary}

The charm production in photon-photon collisions is measured by detecting high momentum leptons. Combining the inclusive electron and muon events, the measured cross section is more than 1.8 standard deviations larger than the incoherent sum of the predictions for the direct process and the photon-gluon fusion process. Here, the mass of the charm quark is assumed to be $1.6 \mathrm{GeV}$ and the statistical and systematic errors are combined in quadrature. The excess cannot be accounted for by tuning the gluon density in the photon over a reasonable range of values.

\section{Acknowledgement}

We thank the TRISTAN staff for the excellent operation of the storage ring. We acknowledge the strong support provided by the staffs of our home institutions. We would like to thank Dr. M. Drees, for valuable discussion on the higher order QCD correction for the cross section of charm production. We would like to thank Dr. K. Hagiwara for providing us the program to calculate the WHIT parton density. This work has been supported by the Japan Ministry of Education, Science and Culture ( Monbusho ), the Japan Society for the Promotion of Science, the US Department of Energy, the US National Science Foundation, the Korean Science and Engineering Foundation, the Ministry of Education of Korea, and the Academia Sinica of the People's Republic of China. 


\section{References}

[1] TASSO Collab., W.Braunschweig et al., Z. Phys. C47 (1990) 499; TPC/2-Gamma Collab., M.Alston-Garnjost et al., Phys. Lett. B252 (1990) 499.

[2] M.Drees, M.Krämer, J.Zunft, and P.M.Zerwas, Phys. Lett. B306 (1993) 371.

[3] S.J.Brodsky, T.A.DeGrand, J.F.Gunion, and J.H.Weis, Phys. Rev. Lett. 41 (1978) 672; Phys. Rev. D19 (1979) 1418;

H.Terazawa, J. Phys. Soc. Jpn. 47 (1979) 355;

K.Kajantie and R.Raitio, Nucl. Phys. B159 (1979) 528;

M.Drees and R.M.Godbole, Nucl. Phys. B339 (1990) 355.

[4] VENUS Collab., S.Uehara et al, Z. Phys. C63 (1994) 213.

[5] TOPAZ Collab., M.Iwasaki et al, Phys. Lett. B341 (1994) 99.

[6] TOPAZ Collab., R.Enomoto et al, Phys. Rev. D50 (1994) 1879;

TOPAZ Collab., R.Enomoto et al, Phys. Lett. B328 (1994) 535.

[7] B.Margolis, Phys. Rev. D17 (1978) 1310;

J.J.Sakurai and D.Schildknecht, Phys. Lett. B40 (1972) 121;

I.F.Ginzburg and V.G.Selbo, Phys. Lett. B109 (1982) 231.

[8] S.Kawabata, Comput. Phys. Commun. 41 (1986) 127.

[9] M. Kuroda, Meiji Gakuen Univ. Research J. 27 (1988) 424.

[10] H.Abramowicz, K.Charchula, and A.Levy, Phys. Lett. B269 (1991) 458.

[11] M.Drees and K.Grassie, Z. Phys. C28 (1985) 451.

[12] K.Hagiwara, M.Tanaka. I.Watanabe, and T.Izubuchi, Phys. Rev. D51 (1995) 3197.

[13] T.Sjöstrand, CERN-TH-6488-92 (May 1992);

T.Sjöstrand and M.Bengtsson, Comput. Phys. Commun. 43 (1987) 367.

[14] C.Peterson, D.Schlatter, I.Schmitt, and P.M.Zerwas, Phys. Rev. D27 (1983) 105.

[15] ALEPH Collab., D.Decamp et al., Phys. Lett. B266 (1991) 218.

[16] Review of Particle Properties, Phys. Rev. D50 (1994) 1172.

[17] AMY Collab., R.Tanaka et al., Phys. Lett. B277 (1992) 215;

AMY Collab., B.J.Kim et al., Phys. Lett. B325 (1994) 248.

[18] AMY Collab., T.Kumita et al., Phys. Rev. D42 (1990) 1339;

AMY Collab., F.Liu et al., Phys. Rev. D49 (1994) 4339.

[19] F.A.Harris et al., Nucl. Instrum. Methods. 103 (1972) 345.

[20] A.Fassò et al., Nucl. Instrum. Methods. A332 (1993) 459;

P.A.Aarnio et al., Proceedings of :1C93 (1993) 88.

[21] Private communication with M.Drees, M.Krämer, and J.Zunft. 


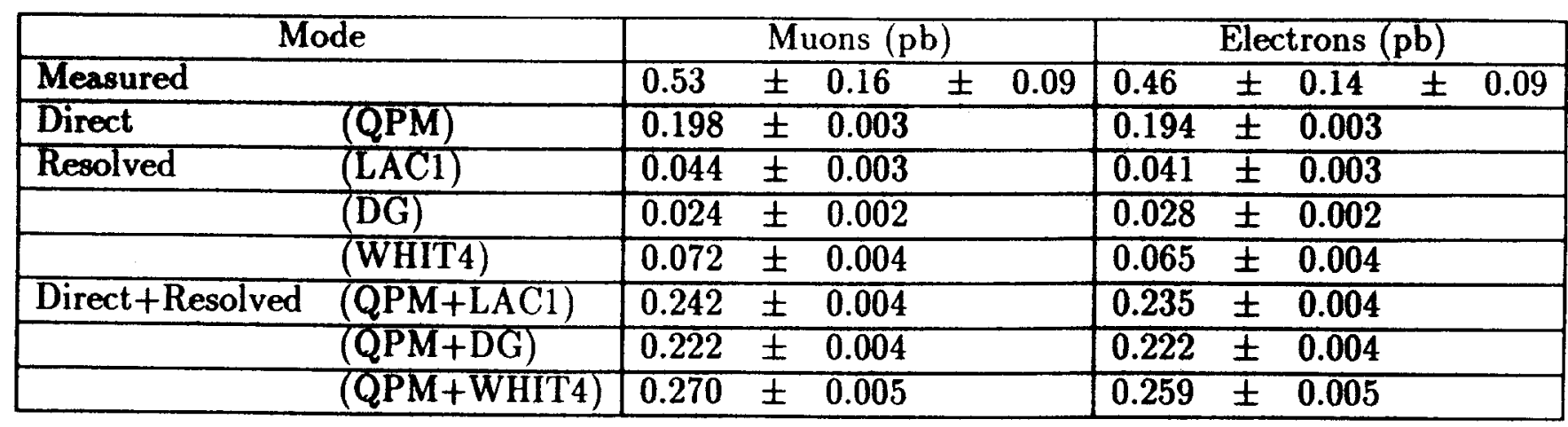

Table 1. Cross sections for inclusive leptons from charm $\sigma\left(e^{+} e^{-} \rightarrow e^{+} e^{-} l^{ \pm} X\right)$ measured by this experiment and theoretical predictions with a charm quark mass of $1.6 \mathrm{GeV}$, including NLO QCD correction.

\begin{tabular}{|ll|lll|lll|}
\hline \multicolumn{2}{|c|}{ Mode } & \multicolumn{3}{|c|}{ Muons (pb) } & \multicolumn{3}{c|}{ Electrons (pb) } \\
\hline Direct & (QPM) & 0.219 & \pm & 0.005 & 0.205 & \pm & 0.005 \\
\hline Resolved & (LAC1) & 0.058 & \pm & 0.008 & 0.062 & \pm & 0.008 \\
\hline & (WHIT4) & 0.076 & \pm & 0.004 & 0.076 & \pm & 0.005 \\
\hline Direct+Resolved & (QPM+LAC1) & 0.277 & \pm & 0.009 & 0.267 & \pm & 0.009 \\
\hline & (QPM+WHIT4) & 0.295 & \pm & 0.006 & 0.281 & \pm & 0.007 \\
\hline
\end{tabular}

Table 2. Cross sections for inclusive leptons from charm $\sigma\left(e^{+} e^{-} \rightarrow e^{+} e^{-l^{ \pm}} X\right)$ predicted theoretically with a charm quark mass of $1.3 \mathrm{GeV}$, including NLO QCD correction. 


\section{List of Figures}

1 Fake muon estimation. (a) The maximum matching distance of the MUO hits with an extrapolated CDC track is set to $1 \mathrm{~m}$ in Monte Carlo simulation. The vertical bar shows the estimation of punchthrough (hatched area) and decay (shaded area) from analytical calculation and the open square shows the Monte Carlo prediction. (b) The maximum matching distance is set to two standard deviations. The open square shows the Monte Carlo prediction. . . . . . . . . . . . .

2 Observed number of leptons with background contributions. Error bar is statistical only. (a) For muons. The closed circle is from the experimental data. The background contributions are $e^{+} e^{-} \rightarrow e^{+} e^{-} \tau^{+} \tau^{-}$ (closed area), one $\gamma$ annihilation multihadronic events (cross-hatched area), $e^{+} e^{-} \rightarrow e^{+} e^{-} b \bar{b}$ (singly-hatched area), and fake muon events (open area). (b) For electrons. The closed circle is from the experimental data. The background contributions are $e^{+} e^{-} \rightarrow e^{+} e^{-} \tau^{+} \tau^{-}$ (closed area), one $\gamma$ annihilation multihadronic events (cross-hatched area), $e^{+} e^{-} \rightarrow e^{+} e^{-b \bar{b}}$ (singly-hatched area), Dalitz pair and $\gamma$ conversion (shaded area), and fake electron events (open area). . . . . . . .

3 Comparison of FLUKA Monte Carlo with experimental data for $E / p$ distributions of different momentum region in annihilation process. The closed circle shows the experimental data and the open square shows the FLUKA prediction. The left and right sides show before and after the shower profile cuts, respectively. The momentum regions are (a) $2.0 \leq p<2.5 \mathrm{GeV} / c$, (b) $2.5<p<4.0 \mathrm{GeV} / c$, and (c) $4.0 \leq p \leq 5.0$ $\mathrm{GeV} / c$.

4 Correlation plot between the transverse momentum of selected leptons and that of $\mathrm{D}$-mesons $\left(D^{*}, D, D_{s}^{*}\right.$, and $\left.D_{s}\right)$ for (a) muons and (b) electrons. These plots were made by using Monte Carlo simulation of the direct and resolved photon processes; $\mathrm{LACl}$ parametarization was used for the gluon density.

5 Cross section of inclusive leptons from charm. The closed circle shows the experimental data. The cross-hatched area shows the prediction for direct process. The singly-hatched area shows the prediction for the photon-gluon fusion process with WHIT4 parton density, where the charm quark mass is assumed to be $1.6 \mathrm{GeV}$. The open area shows the increase of cross section due to the lighter charm quark mass of $1.3 \mathrm{GeV}$. The NLO QCD correction is included in these predictions. The error bar is the combined statistical and systematic uncertainty. . . . . . . 


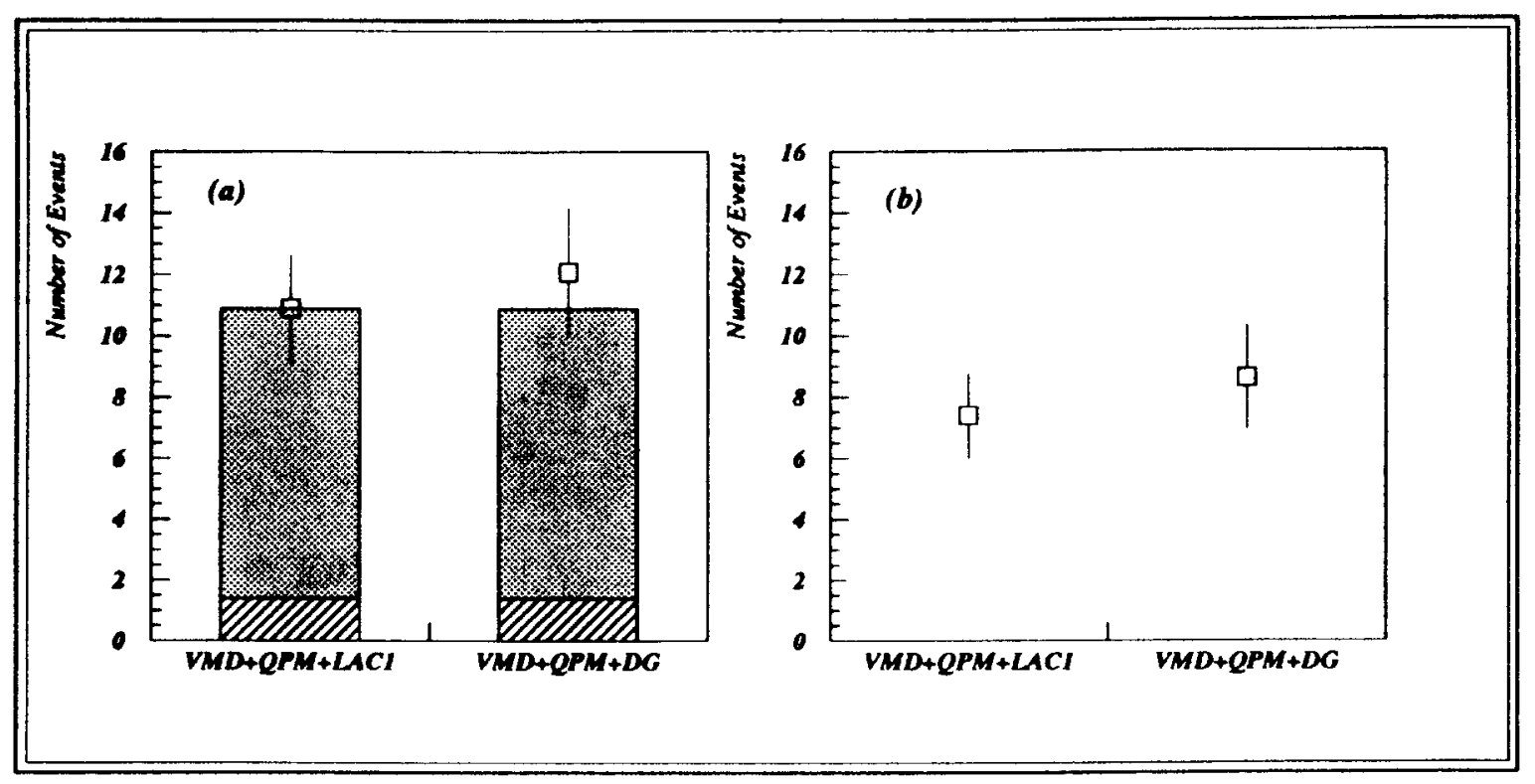

Fig.1. Fake muon estimation. (a) The maximum matching distance of the MUO hits with an extrapolated CDC track is set to $1 \mathrm{~m}$ in Monte Carlo simulation. The vertical bar shows the estimation of punchthrough (hatched area) and decay (shaded area) from analytical calculation and the open square shows the Monte Carlo prediction. (b) The maximum matching distance is set to two standard deviations. The open square shows the Monte Carlo prediction.

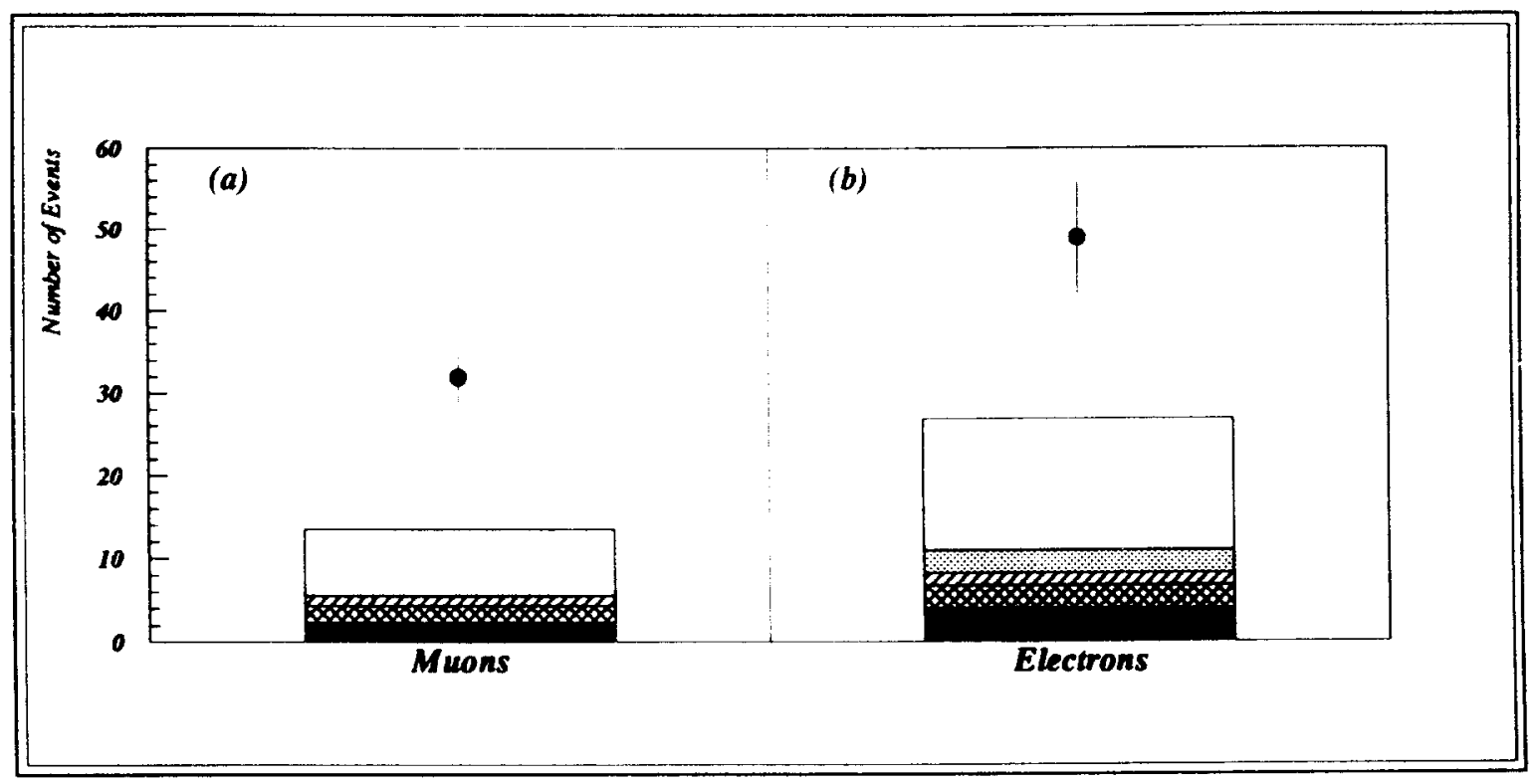

Fig.2. Observed number of leptons with background contributions. Error bar is statistical only. (a) For muons. The closed circle is from the experimental data. The background contributions are $e^{+} e^{-} \rightarrow e^{+} e^{-} \tau^{+} \tau^{-}$(closed area), one $\gamma$ annihilation multihadronic events (cross-hatched area), $e^{+} e^{-} \rightarrow e^{+} e^{-b \bar{b}}$ (singly-hatched area), and fake muon events (open area). (b) For electrons. The closed circle is from the experimental data. The background contributions are $e^{+} e^{-} \rightarrow e^{+} e^{-} \tau^{+} \tau^{-}$ (closed area), one $\gamma$ annihilation multihadronic events (cross-hatched area), $e^{+} e^{-}-e^{+} e^{-b \bar{b}}$ (singlyhatched area), Dalitz pair and $\gamma$ conversion (shaded area), and fake electron events (open area). 


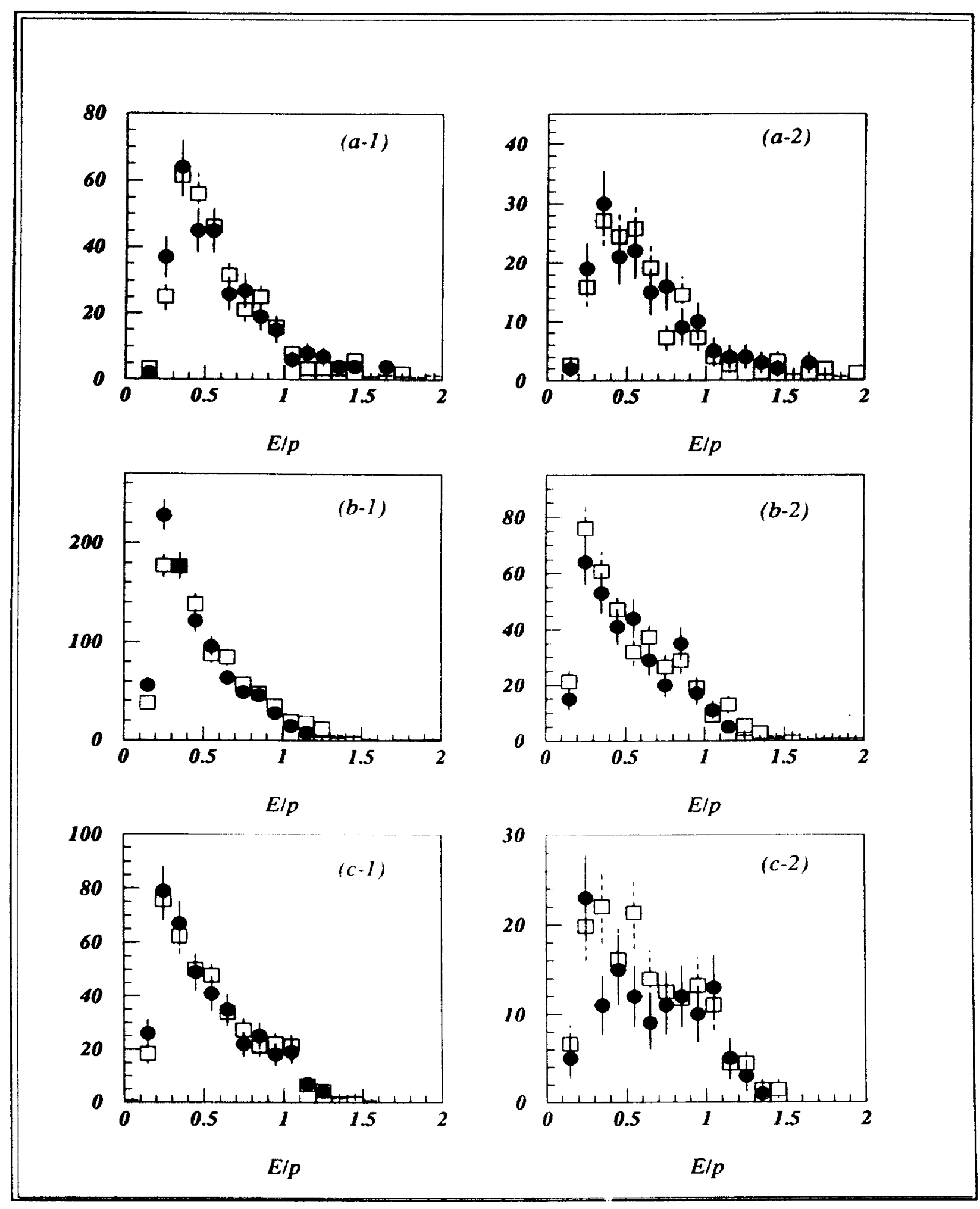

Fig.3. Comparison of FLUKA Monte Carlo with experimental data for $E / p$ distributions of different momentum region in annihilation process. The closed circle shows the experimental data and the open square shows the FLUKA prediction. The left and right sides show before and after the shower profile cuts, respectively. The momentum regions are (a) $2.0 \leq p<2.5 \mathrm{GeV} / c$, (b) $2.5 \leq p<4.0$ $\mathrm{GeV} / c$, and (c) $4.0 \leq p \leq 5.0 \mathrm{GeV} / c$. 


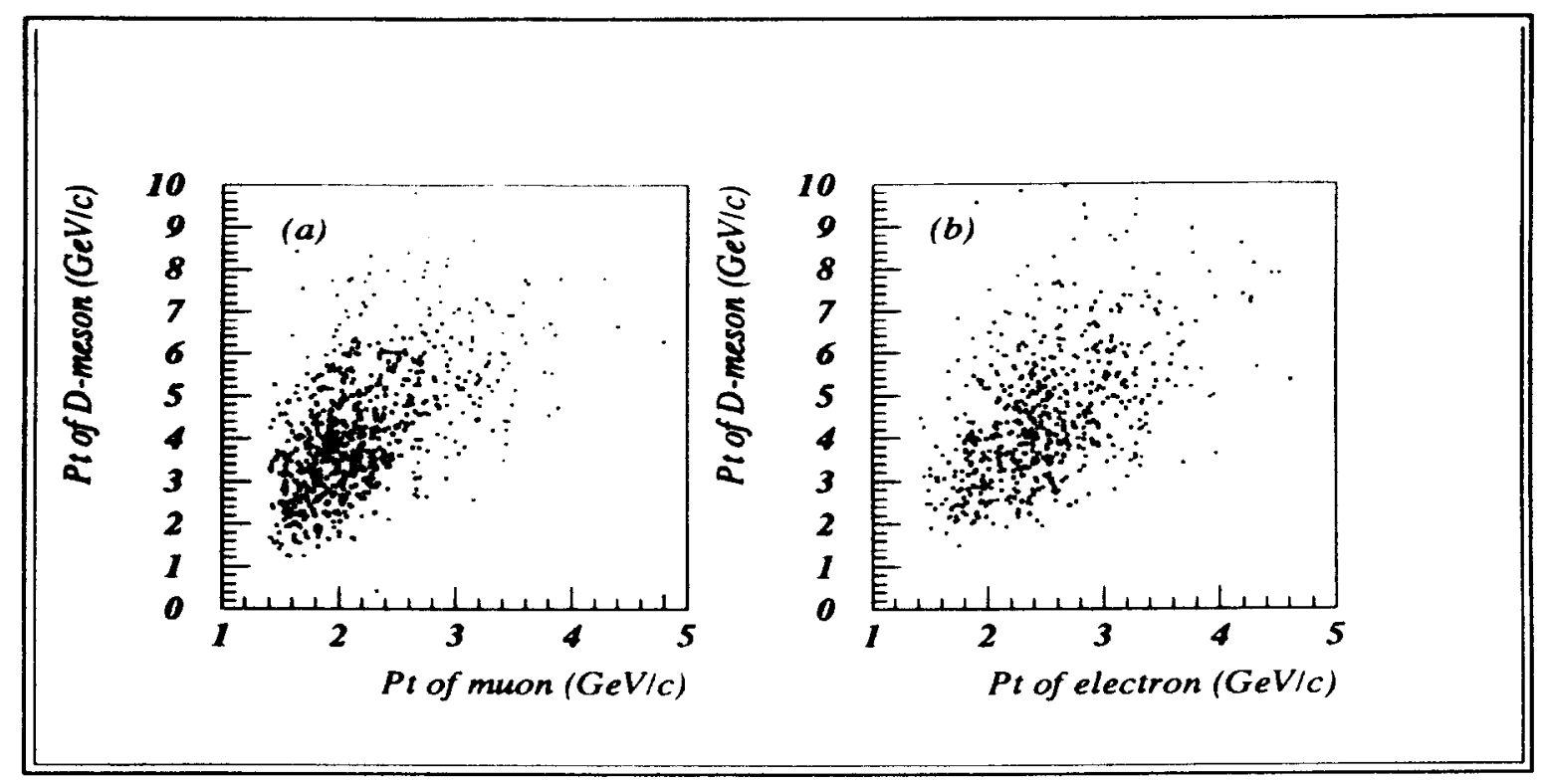

Fig.4. Correlation plot between the transverse momentum of selected leptons and that of Dmesons $\left(D^{*}, D, D_{s}^{*}\right.$, and $\left.D_{3}\right)$ for (a) muons and (b) electrons. These plots were made by using Monte Carlo simulation of the direct and resolved photon processes; LAC1 parametarization was used for the gluon density.

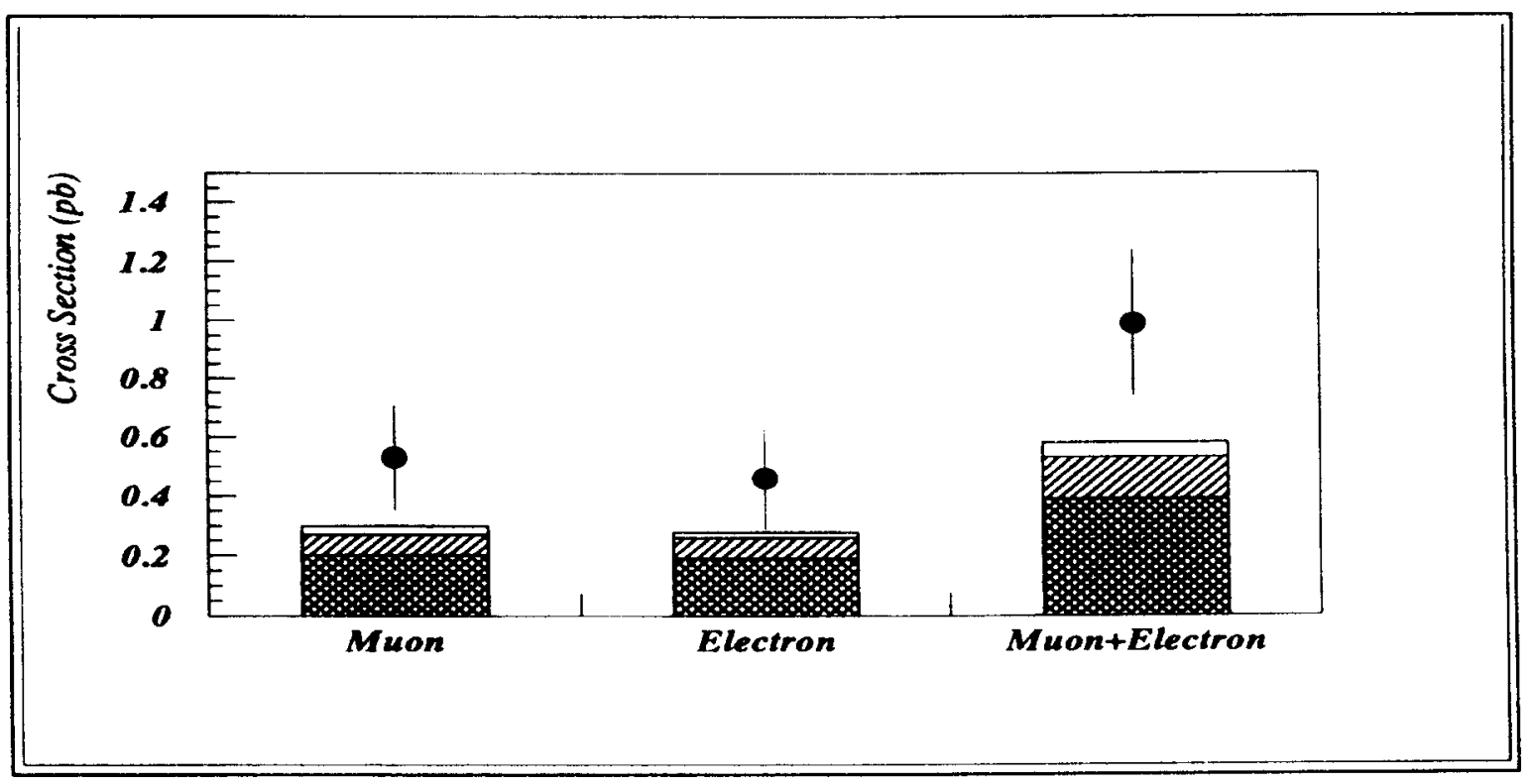

Fig.5. Cross section of inclusive leptons from charm. The closed circle shows the experimental data. The cross-hatched area shows the prediction for direct process. The singly-hatched area shows the prediction for the photon-gluon fusion process with WHIT4 parton density, where the charm quark mass is assumed to be $1.6 \mathrm{GeV}$. The open area shows the increase of cross section due to the lighter charm quark mass of $1.3 \mathrm{GeV}$. The NLO QCD correction is included in these predictions. The error bar is the combined statistical and systematic uncertainty. 